\title{
Intrathecally Expanding B Cell Clones in Herpes Simplex Encephalitis: A Case Report
}

\author{
Shila P. Coronel-Castello • Gildas Lepennetier · Jolien Diddens • \\ Verena Friedrich · Monika Pfaller · Bernhard Hemmer • \\ Klaus Lehmann-Horn
}

Received: November 23, 2021 / Accepted: January 24, 2022 / Published online: February 6, 2022

(c) The Author(s) 2022

\begin{abstract}
Introduction: In spite of antiviral treatment, herpes simplex encephalitis (HSE) remains associated with a poor prognosis and often results in neurological impairment. The $\mathrm{B}$ cell response in HSE is poorly understood. The objective of this study was to identify, in a patient with HSE, B cell clones in cerebrospinal fluid (CSF) cells and peripheral blood mononuclear cells (PBMCs) that expanded between two different time points during the course of infection.

Methods: CSF cells and PBMCs were sampled from a HSE patient at two time points 5 days
\end{abstract}

Shila P. Coronel-Castello and Gildas Lepennetier contributed equally to this work.

Supplementary Information The online version contains supplementary material available at https:// doi.org/10.1007/s40120-022-00330-2.

S. P. Coronel-Castello · G. Lepennetier · J. Diddens

. V. Friedrich - M. Pfaller - B. Hemmer .

K. Lehmann-Horn $(\bowtie)$

Department of Neurology, School of Medicine,

Technical University of Munich, Munich, Germany

e-mail: klaus.lehmann-horn@tum.de

B. Hemmer

Munich Cluster of Systems Neurology (SyNergy),

Munich, Germany apart. B cells were analyzed using single-cell immune profiling (CSF cells) and conventional deep immune repertoire sequencing (PBMCs).

Results: We identified CSF B cell clones that expanded from time 1 to time 2 . Some of these $B$ cell clones could also be found in the peripheral blood. We also report the corresponding B cell receptor (BCR) sequences.

Conclusion: In our patient, HSE resulted in an intrathecal B cell response with expanding CSF clones. We report the B cell receptor sequences of several expanding and dominating clones; these sequences can be used to create recombinant antibodies. Even though the antigen specificity of these expanding clones is unknown, our findings suggest that an adaptive immune response in the central nervous system contributes to repelling herpes simplex virus infection in the brain.

Keywords: Herpes simplex encephalitis; B cell repertoire; Intrathecal B cell response; Singlecell sequencing; Case report 


\section{Key Summary Points}

A single case report on intrathecally expanding B cell clones in a 28 -year-old female patient with herpes simplex encephalitis is provided.

We report the B cell receptor sequences of expanding and dominating B cell clones in the cerebrospinal fluid.

These findings can be used for further studies and may potentially lead to the generation of neutralizing recombinant antibodies.

\section{INTRODUCTION}

Herpes simplex encephalitis (HSE) caused by herpes simplex virus (HSV) represents $5-15 \%$ of all infectious encephalitis cases [1]. HSE results in inflammation and necrosis, mostly in the medial-temporal lobes and orbital-frontal regions of the brain [2]. It has been postulated that the infection of the brain originates from a latent infection of the trigeminal ganglion or through infiltration via the nose [3]. Under antiviral therapy, mortality is about $20-30 \%$, and residual neurological impairment persists in $38-56 \%$ of patients [4].

$\mathrm{T}$ cells, B cells, and other leukocytes were found in parenchymal and meningeal infiltrates [5], and the $\mathrm{T}$ cell immune response has been studied [6]. For instance, analysis of the $\mathrm{T}$ cell repertoire in murine trigeminal ganglia infected with HSV-1 revealed that the most widely recognized epitope was viral glycoprotein B ( $\mathrm{gB}$ 498-505) [3].

B cell studies showed a persistent intrathecal presence of specific IgG many years after HSE or in spite of negative PCR results during acute infection $[7,8]$. A specific IgG index demonstrated intrathecal production of HSV-1 IgG, which persisted two years after clinical onset [9]. Similarly, HSV-1-specific oligoclonal IgG bands were found in the cerebrospinal fluid (CSF). However, in this study, and in contrast to the previously cited study, they persisted in the CSF for only a limited period of time, but they persisted longer in the serum [10].

B cell repertoire sequencing has been performed in various forms of autoimmune neurological disorders, such as multiple sclerosis and anti- $N$-methyl-D-aspartate receptor encephalitis [11, 12], but, to our knowledge, not in HSE. Studies of viral encephalitis caused by other viruses have shown the potential of investigating the $\mathrm{B}$ cell repertoire in these diseases. For example, single-cell analysis by microengraving and next-generation sequencing (NGS) of peripheral blood mononuclear cells (PBMCs) from West Nile virus (WNV) encephalitis patients identified WNV-specific neutralizing antibodies [13]. A recent report that used NGS for peripheral blood B cell receptor (BCR) and T cell receptor (TCR) analysis of patients with multiple sclerosis on immunosuppressive therapy who developed progressive multifocal leukoencephalopathy (PML) caused by the John Cunningham (JC) virus showed that no JCV-related specific T- and B-cell expansions were mounted at the time of PML [14]. This may be related to the opportunistic nature of PML, which occurs in immunosuppressed hosts.

The objective of this study was to analyze the B cell repertoire from a patient with HSE at two different time points. We found clonal expansion in the CSF, suggesting an intrathecal immune response.

\section{METHODS}

We investigated the B cell repertoire of a patient with HSE who was treated in the Department of Neurology at Technical University of Munich School of Medicine university hospital in 2009. All patient-related data were extracted from the electronic record. CSF and PBMC samples were collected and frozen in liquid nitrogen in 2009 and analyzed between 2019 and 2021. The patient's agreement and consent to participate and for publication were obtained within the framework of the Biobank of the Department of Neurology as part of the Joint Biobank Munich in the framework of the German Biobank Node. 
This study was approved by the local ethics committee (Ethics Committee of the TUM School of Medicine, Munich, Germany); reference number 58/19 S. This study was performed in accordance with the Helsinki Declaration of 1964 and its later amendments.

\section{Description of the Case}

The 28-year-old female patient presented with headache, fevers, behavioral changes, decreased consciousness, and left hemiparesis for three days. The cranial MRI showed a T2/FLAIR hyperintensity, particularly in the right temporal lobe with leptomeningeal gadolinium enhancement (Fig. 1). The initial CSF analysis showed pleocytosis (292 cells/ $\mu \mathrm{l})$; HSV-1 was detected in the CSF by PCR $(411,000$ genome equivalents $/ \mathrm{ml}$ ). No CSF cells were collected from this initial lumbar puncture. The patient was initially treated with acyclovir $750 \mathrm{mg}$ i.v. every $8 \mathrm{~h}$. After mild clinical improvement, the patient developed a vertical gaze palsy 5 days after the beginning of treatment. An epileptic seizure occurred and was treated with levetiracetam. Electroencephalography showed isolated sharp waves in the right hemisphere, and a follow-up MRI showed an expansion of FLAIR hyperintensity in the right temporal and parietal lobe. The second CSF analysis, 5 days after the first lumbar puncture, showed 338 cells/ $\mu \mathrm{l}$ and an increased viral load (1.4 Mio. genome equivalents/ml). Assuming acyclovir resistance, treatment was changed to foscarnet $40 \mathrm{mg} / \mathrm{kg}$ bodyweight i.v. every $8 \mathrm{~h}$, which led to partial recovery with a residual headache and vertical gaze palsy within 7 days. A third CSF analysis 5 days after the second showed reduced pleocytosis ( 76 cells/ $\mu \mathrm{l}$ ), and no HSV genes were detected by PCR. The patient was discharged 13 days after the last lumbar puncture for rehabilitation in her home country. CSF cell pellets and PBMCs extracted from peripheral blood samples at the second lumbar puncture (first time point, t1) and at the third lumbar puncture (second time point, $\mathrm{t} 2$ ) were stored in liquid nitrogen for further analysis.

\section{Design}

CSF cells at $\mathrm{t} 1$ and $\mathrm{t} 2$ were analyzed using a single-cell immune profiling technology. PBMCs at $\mathrm{t} 1$ and $\mathrm{t} 2$ were analyzed using a conventional immune profiling approach featuring bulk sequencing of immunoglobulin (Ig) heavy chains. We determined the expanding CSF B cell clones and the overlap with the peripheral blood B cell repertoire.

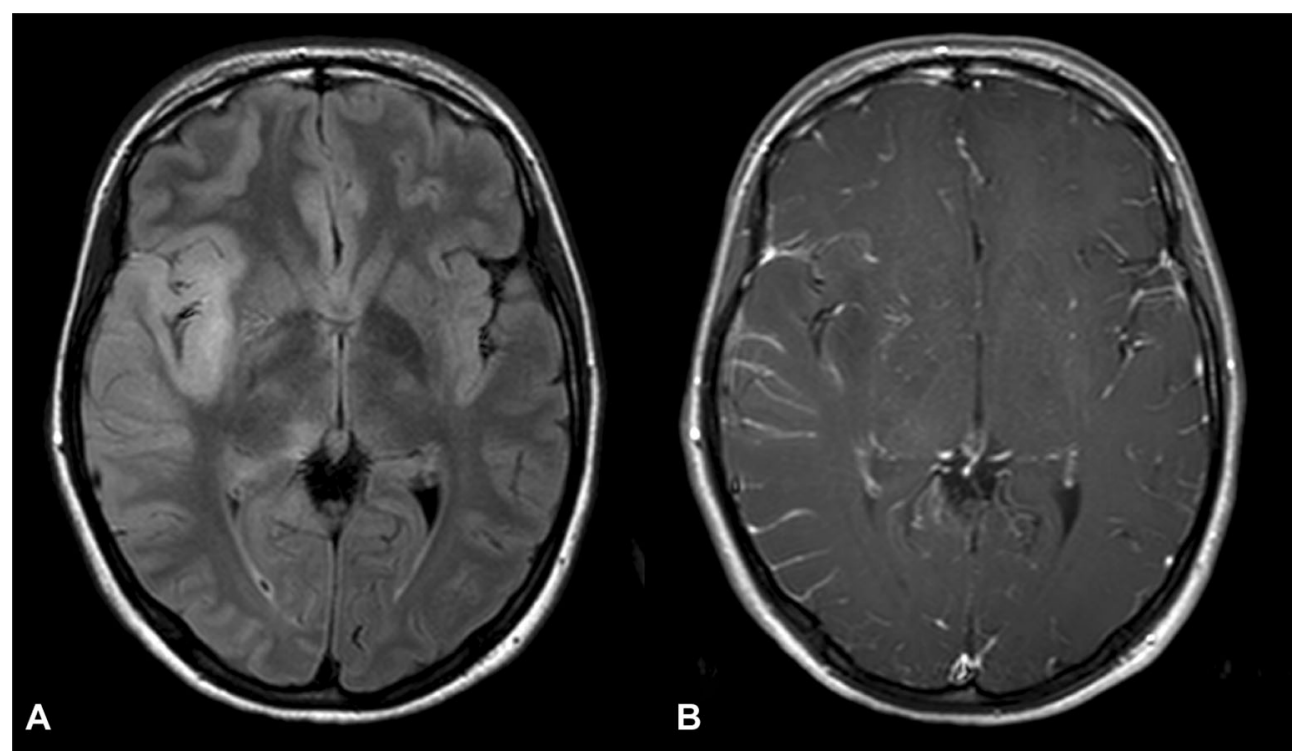

Fig. 1 Cerebral MRI of a patient with HSE. Initial MRI was obtained 1 day after hospitalization, with T2/FLAIR (A) and gadolinium contrast-enhanced T1-weighted sequences $(\mathbf{B})$. This image was taken from the patient's electronic files 


\section{Single-Cell BCR Sequencing of CSF Samples}

After thawing and washing the cryopreserved $\mathrm{t} 1$ and $\mathrm{t} 2$ CSF samples, a single-cell suspension was prepared using the $10 \times$ Genomics Sample Preparation protocol (CG00039, Rev C). Cell count and viability were measured using an automated cell counter (Cellometer Auto 2000, Nexcelom Bioscience), and the volume was adjusted to obtain $1 \times 10^{6}$ cells $/ \mathrm{ml}$. Cells were loaded onto a $10 \times$ Genomics Chromium chip per the manufacturer's recommendations, with a targeted cell recovery of $1 \times 10^{4}$ cells. Reverse transcription and library preparation was performed using the $10 \times$ Genomics Chromium Single Cell Immune Profiling (v1) kit, following the $10 \times$ Genomics protocol (CG000086, Rev J). After quantitative PCR-based library quantification using primers targeting the p5 and p7 (p5: AATGATACGGCGACCACCGAGAT,

p7: CAAGCAGAAGACGGCATACGA) regions of Illumina adapters and KAPA Library Quantification Standards (KK4903, Kappy Biosystems), single-cell BCR V(D)J libraries were prepared using the MiSeq Reagent Kit v2 (300-Cycles, Illumina) and sequenced on a Miseq sequencing platform (Miseq Control Software, version 2.6.2., Illumina) with paired-end reads $(151 \times 151 \mathrm{bp})$.

\section{Bulk BCR Sequencing of PBMCs}

PBMC samples from $\mathrm{t} 1$ and $\mathrm{t} 2$ were thawed and RNA was extracted using the RNeasy ${ }^{\circledR}$ Mini Kit (Qiagen). cDNA libraries were prepared using the Takara SMARTer ${ }^{\circledR}$ Human BCR IgG IgM $\mathrm{H} / \mathrm{K} / \mathrm{L}$ Profiling Kit (Takara) according to the manufacturer's protocol. Following quantification using quantitative PCR, the library was prepared using the MiSeq Reagent Kit v3 (600Cycles, Illumina) sequenced on a Miseq platform (Illumina) with paired-end reads $(301 \times 301 \mathrm{bp})$.

\section{Bioinformatics}

The CSF B cell libraries (single-cell data) were processed using Cell Ranger (v3.1.0,
$10 \times$ Genomics) and aligned with human V(D)J reference (vdj_GRCh38_alts_ensembl-3.1.03.1.0). The libraries of PBMC samples (bulk sequencing data) were processed with Immune Profiler, modifying the external library to use the latest version of the tool mixcr-3.0.12. Only productive full-length sequences were considered for analysis. After quality filtering (excluding wrong cell types and clonotypes with no V-gene match), we focused only on paired chains. Overlapping B cell clonotypes (based on the same complementary determining region (CDR) 3 nucleotide sequence) between $\mathrm{t} 1$ and $\mathrm{t} 2$ were selected. We created new clonal groups using a hamming distance of $\leq 1$ between CDR3 amino acid sequences and $\mathrm{V}$ and $\mathrm{J}$ gene identity. We selected the most expanded clones (based on the ratio $t 2 / t 1$ ) using the percentage instead of the cell count to correct for sample size bias. Full-length sequences of the chains were extracted from the $10 \times$ data and re-analyzed using the IMGT HighVQuest online tool [15].

\section{RESULTS}

\section{Analysis of Overlap in B Cell Repertoires Reveals Greater Temporal Changes in CSF Compared to PBMCs}

We looked for B cell clones based on the CDR3 nucleotide sequence (Fig. 2). In the CSF sample from t1 (1964 B cells) we found 1354 clones, and in the CSF sample from t2 (333 cells) we found 260 clones. Among the clones in these two groups, 76 (4.9\% of all clones found in CSF) overlapped between $\mathrm{t} 1$ and $\mathrm{t} 2$. In the PBMCs from $\mathrm{t} 1$ ( $2476 \mathrm{~B}$ cells) we found 329 clones, and at t2 (1867 B cells) there were 340 clones. Among the clones in these two groups, 127 (23.4\% of all clones in PBMCs) overlapped. This suggests a greater change over time (5 days) in the CSF than in PBMCs. However, it has to be taken into consideration that we applied different sequencing techniques for CSF and PBMC samples, which may represent a limitation. Moreover, upon comparing CSF and PBMCs, we found 71 overlapping clones at $\mathrm{t} 1$ (4.4\% of all clones; $3.6 \%$ overlap of CSF B cells 

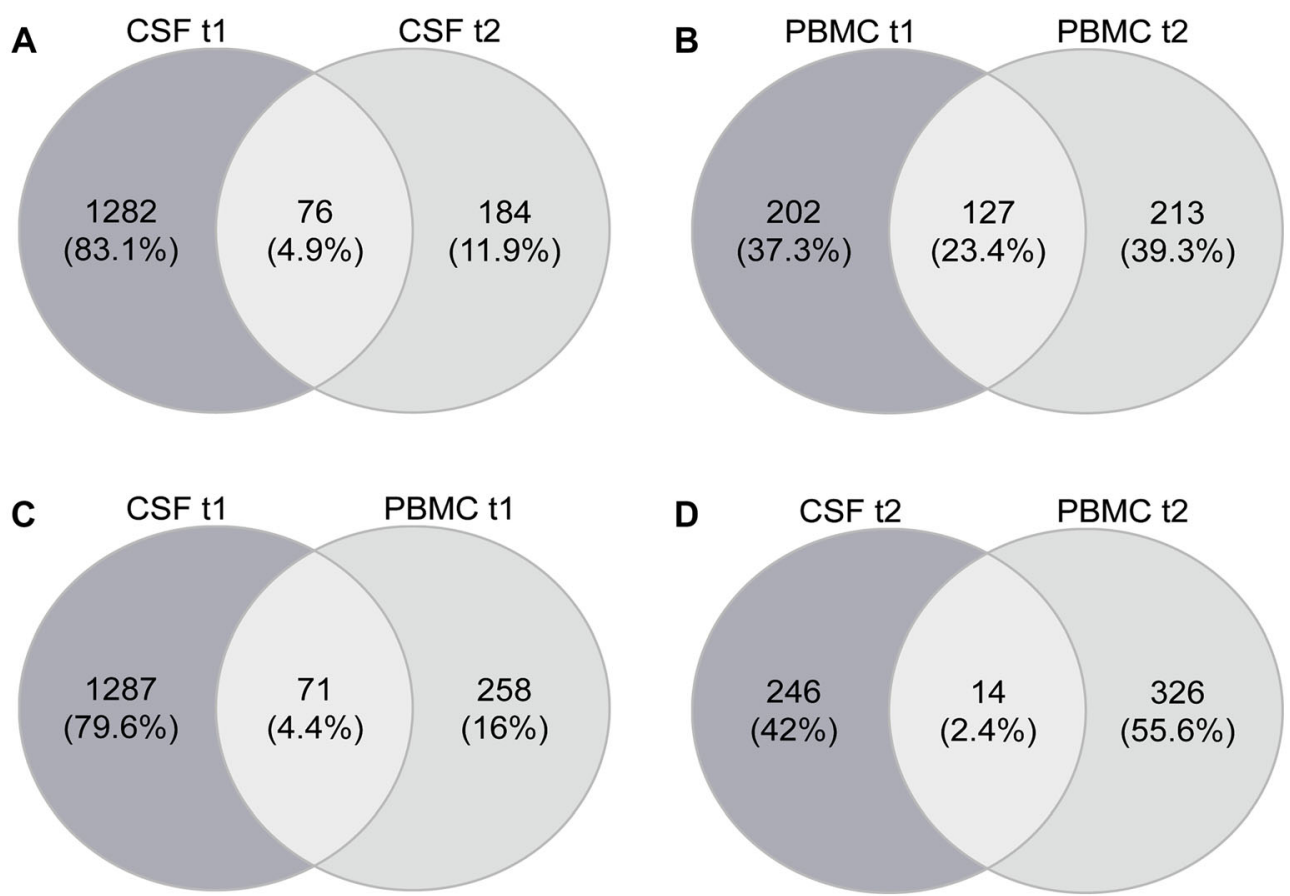

Fig. 2 Analysis of overlap in B cell repertoires. Venn diagrams showing the overlapping $\mathrm{B}$ cell clones based on the CDR3 nucleotide sequence in CSF cells at $t 1$ versus $t 2$

(A), in PBMCs at $\mathrm{t} 1$ versus $\mathrm{t} 2$ (B), as well as in CSF cells at $\mathrm{t} 1$ versus PBMCs at $\mathrm{t} 1(\mathbf{C})$, and in CSF cells at $\mathrm{t} 2$ versus PBMCs at t2 (D)

with peripheral B cells) and 14 overlapping clones at $\mathrm{t} 2$ (2.4\% of clones; $4.2 \%$ overlap of CSF $B$ cells with peripheral B cells).

\section{Identification of Clonal Groups That Expanded from $t 1$ to $t 2$}

We created clonal groups using a hamming distance of $\leq 1$ between CDR3 amino acid sequences. We focused on the clonal groups that showed the greatest expansion based on the ratio between $t 2$ and $t 1$ (Fig. 3). Since the cell counts at $\mathrm{t} 1(n=1964$ cells $)$ and $\mathrm{t} 2(n=333$ cells) were different, we calculated the ratio as the percentage of cells in one clonal group at $\mathrm{t} 2$ in relation to the percentage of cells in the same clonal group at $\mathrm{t} 1$. We found 87 clonal groups with a ratio above 1 (i.e., expanding clonal groups) and 4 clonal groups with a ratio below 1. Expanding clonal groups of CSF B cells suggest an ongoing intrathecal $B$ cell response between $t 1$ and $t 2$. The mean expansion ratio

was 4.7 and the median of the expansion ratio was 5.9. Among 87 clonal groups with expansion ratios $\geq 1$, we found 16 that overlapped with the BCR repertoire of PBMCs. The CDR3 amino acid sequences of a selection of interesting clonal groups are shown in Table 1, including information about isotype and $\mathrm{V}(\mathrm{D}) \mathrm{J}$ gene usage. This selection includes the six clones with the highest expansion ratio (11.8), two clones (nos. 6 and 8 ) with a high expansion ratio that were present in a high amount of $B$ cells, and two clones (nos. 141 and 53) that were also a dominant presence in $\mathrm{B}$ cells but had lower expansion ratios. This table also shows two clones (nos. 14 and 25) with high expansion ratios that overlapped with PBMCs, and clone no. 2, which was present in a high percentage of the cells at both $\mathrm{t} 1$ and $\mathrm{t} 2$, but presented a limited expansion ratio. Supplementary Table 1 provides the full nucleotide sequences of CDR3 for all the clones/clonal groups with expansion ratios $\geq 1$. 


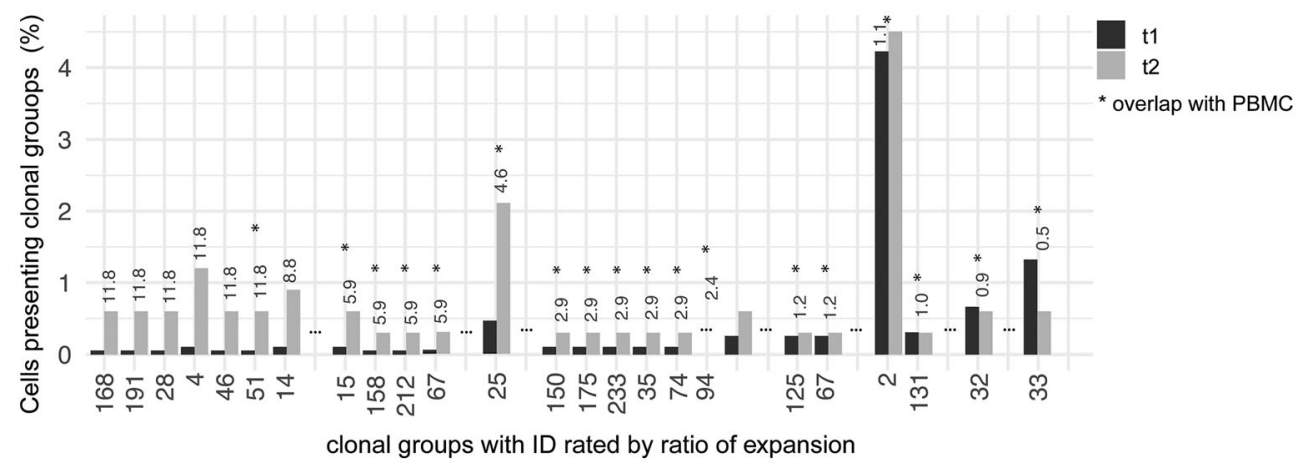

Fig. 3 Identification of CSF clonal groups that expanded from $t 1$ to $t 2$. B cell clonal groups are ranked according to the ratio of expansion between $\mathrm{t} 2$ (gray bars) and $\mathrm{t} 1$ (black bars). This figure shows the percentage of cells representing one clonal group among all the cells at $\mathrm{t} 1$ or $\mathrm{t} 2$; the clonal group ID is the number below the bars. The ratio number above each clonal group) was calculated as the percentage of cells representing one clonal group among all CSF cells at

\section{DISCUSSION}

It is known that an intrathecal humoral immune response, represented by CSF HSV-1 specific oligoclonal bands and an elevated IgG index, occurs in and after HSE [9]. Utilizing a single-cell-based B cell repertoire sequencing approach, we complemented these previous findings by demonstrating an expansion of B cell clones in the CSF in the acute and early recovery phase in a case of HSE. We provide the nucleotide sequences of these expanded B cell clones, which can be used for further studies.

We obtained two CSF cell samples 5 days apart from a patient with HSV-1 encephalitis with presumed acyclovir resistance, who recovered after switching to foscarnet therapy. The first sample (taken at t1) was obtained after the patient had worsened clinically while being treated with acyclovir during the acute phase of the infection. At this time, 8 days after the first symptoms occurred, the adaptive immune response was assumed to be at an early stage [16]. The second sample (taken at t2) was obtained when the patient had already begun to recover, with the adaptive immune response presumably fully active. Unfortunately, no CSF cells could be preserved from the initial diagnostic CSF puncture. The CSF cell count decreased from $t 1$ to $t 2$, as did the HSV CSF viral t2 in relation to the percentage of cells representing the same clonal group among all CSF cells at t1. Due to the large number of clonal groups, and for clarity, we only include a selection of clonal groups in the figure: those with the top seven ratios and those that overlapped with PBMCs. Clonal groups that overlapped between CSF and PBMCs are marked with an asterisk $\left(^{*}\right)$

load, reflecting the clinical improvement. Importantly, several B cell clones that were found in the CSF repertoires of both samples expanded from t1 to $t 2$. This suggests an intrathecal B cell response, presumably against HSV-1. Our findings contribute to our understanding of the adaptive immune response against HSV-1 infection of the central nervous system (CNS). Potentially, high-affinity virusneutralizing antibodies with therapeutic potential may be identified using this approach.

Besides the fact that it is a report of a single case, an obvious limitation of our study is that we do not know the specificity of the expanded B cell clones. Our findings need to be confirmed in other patients, and specificity towards HSV-1 should be established. Another open question remains whether any putative HSV-1-specific B cells produce neutralizing antibodies.

While CSF samples were analyzed by singlecell sequencing, bulk Ig heavy-chain sequencing was utilized for PBMCs. Although sequence information on the Ig heavy-chain VDJ region is generally sufficient to unequivocally determine B cell clones [17], we are missing information regarding the respective Ig light chain in PBMCs.

Our analysis comparing B cell repertoires from the CSF versus the peripheral blood on the one hand and from $t 1$ versus $t 2$ on the other 


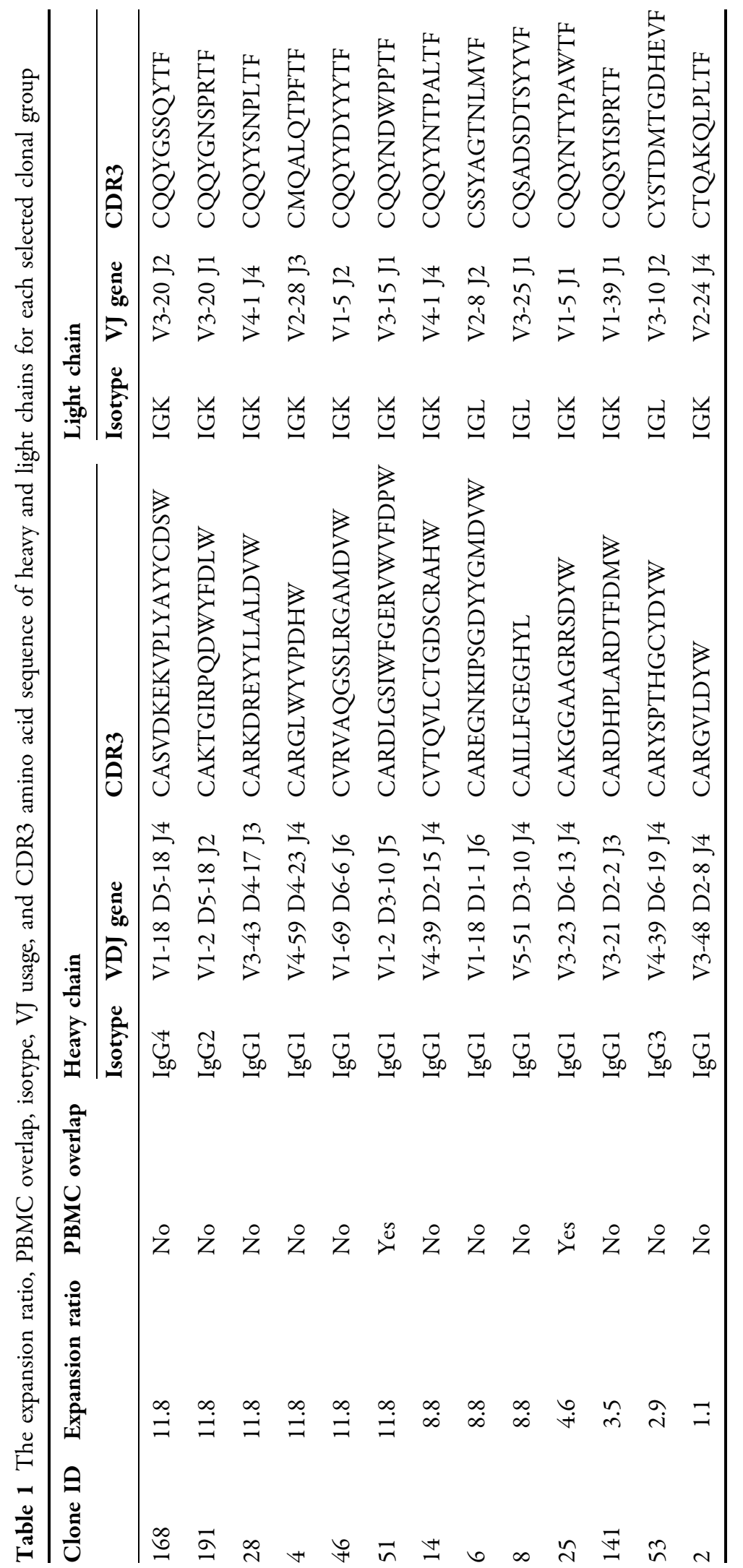


hand demonstrated a relatively small overlap between the respective conditions. Rather than reflecting dramatic differences between the peripheral and CNS immune compartments or dramatic changes over a short period of time (5 days) within them, this is likely due to the sampling of only a small fraction of the entire PBMC or CSF cell pool at $\mathrm{t} 1$ and $\mathrm{t} 2$. We speculate that the $\mathrm{B}$ cell clones that do overlap include the most prevalent clones overall. In the CSF, during an immune response against HSV-1, these presumably include B cells specific for HSV-1. Similarities between the B cell repertoires at $\mathrm{t} 1$ and $\mathrm{t} 2$ were larger in PBMCs than in the CSF, suggesting a substantial and rapid intrathecal immune response during HSE.

\section{CONCLUSION}

We have demonstrated intrathecal expansion of $B$ cell clones in a case of HSE and provided the BCR sequences of the expanding clones. These sequences may be used to create recombinant antibodies and prove HSV specificity.

\section{ACKNOWLEDGEMENTS}

We thank our patient for agreeing to participate in this study. We thank the Biobank team of the Department of Neurology at TUM School of Medicine for collecting and preparing the samples. Klaus Lehmann-Horn received research support from the Deutsche Forschungsgemeinschaft (SFB-TR-128, projects A4 and A12, and LE 3079/3-1) and the Hertie Foundation (MyLab program).

Funding. No funding or sponsorship was received for this study or publication of this article. The Rapid Service Fee was funded by the authors.

Authorship. All named authors meet the International Committee of Medical Journal Editors (ICMJE) criteria for authorship for this article, take responsibility for the integrity of the work as a whole, and have given their approval for this version to be published.
Author Contributions. Shila P. CoronelCastello and Klaus Lehmann-Horn conceived the study. Shila P. Coronel-Castello, Jolien Diddens, Verena Friedrich, Monika Pfaller performed the experiments. Gildas Lepennetier performed the bioinformatic analysis. Shila P. Coronel-Castello, Gildas Lepennetier, Jolien Diddens, Bernhard Hemmer and Klaus Lehmann-Horn interpreted the results. Shila P. Coronel-Castello, Gildas Lepennetier, Jolien Diddens, Bernhard Hemmer and Klaus Lehmann-Horn wrote the manuscript. All authors read and approved the final manuscript.

Disclosures. Shila P. Coronel-Castello, Gildas Lepennetier, Jolien Diddens, Verena Friedrich and Monika Pfaller have nothing to disclose. Bernhard Hemmer has served on scientific advisory boards for Novartis; he has served as DMSC member for AllergyCare, Polpharma and TG therapeutics; he or his institution have received speaker honoraria from Desitin; his institution received research grants from Regeneron for multiple sclerosis research. He holds part of two patents; one for the detection of antibodies against KIR4.1 in a subpopulation of patients with multiple sclerosis and one for genetic determinants of neutralizing antibodies to interferon. All conflicts are not relevant to the topic of the study. All CoI are not related to the content of the project. Klaus Lehmann-Horn has received research support (to TUM) from Novartis, honoraria and compensation for travel expenses from Novartis, F. Hoffmann-La Roche, Biogen, Teva and Merck Serono. All conflicts are not relevant to this study.

Compliance with Ethics Guidelines. The patient's agreement and consent to participate and for publication were obtained within the framework of the Biobank of the Department of Neurology as part of the Joint Biobank Munich in the framework of the German Biobank Node. This study was approved by the local ethics committee (Ethic Committee of the TUM School of Medicine, Munich, Germany) with the reference number 58/19 S. This study was performed in accordance with the Helsinki declaration of 1964 and its later amendments. 
Data Availability. The datasets generated and analyzed during this study, which support the conclusion of this article, are included in this published article and as supplementary information files. Remaining or unprocessed datasets are available from the corresponding author on reasonable request.

Open Access. This article is licensed under a Creative Commons Attribution-NonCommercial 4.0 International License, which permits any non-commercial use, sharing, adaptation, distribution and reproduction in any medium or format, as long as you give appropriate credit to the original author(s) and the source, provide a link to the Creative Commons licence, and indicate if changes were made. The images or other third party material in this article are included in the article's Creative Commons licence, unless indicated otherwise in a credit line to the material. If material is not included in the article's Creative Commons licence and your intended use is not permitted by statutory regulation or exceeds the permitted use, you will need to obtain permission directly from the copyright holder. To view a copy of this licence, visit http://creativecommons.org/licenses/by$\mathrm{nc} / 4.0 /$.

\section{REFERENCES}

1. Rozenberg F. Herpes simplex virus and central nervous system infections: encephalitis, meningitis, myelitis. Virol (Montrouge). 2020;24(5):283-94.

2. Widener RW, Whitley RJ. Herpes simplex virus. Handb Clin Neurol. 2014;123:251-63.

3. St Leger A, Peters B, Sidney J, Sette A, Hendricks R. Defining the herpes simplex virus-specific CD8+ T cell repertoire in $\mathrm{C} 57 \mathrm{BL} / 6$ mice. J Immunol. 2011;186(7):3927-33.

4. Amtage F, Bär M, Bengel G, Bogdan C, Braune S, Brich J, et al. Neurologie compact. Stuttgart: Georg Thieme Verlag; 2017. http://www.thieme-connect. de/products/ebooks/book/10.1055/b-005-143671.

5. Sobel RA, Collins AB, Colvin RB, Bhan AK. The in situ cellular immune response in acute herpes simplex encephalitis. Am J Pathol. 1986;125(2): 332-8.
6. Piret J, Boivin G. Immunomodulatory strategies in herpes simplex virus encephalitis. Clin Microbiol Rev. 2020;33:2.

7. Aurelius E, Forsgren M, Sköldenberg B, Strannegård O. Persistent intrathecal immune activation in patients with herpes simplex encephalitis. J Infect Dis. 1993;168(5):1248-52.

8. Denes $\mathrm{E}$, Labach $\mathrm{C}$, Durox $\mathrm{H}$, et al. Intrathecal synthesis of specific antibodies as a marker of herpes simplex encephalitis in patients with negative PCR. Swiss Med Wkly. 2010;140:w13107.

9. Ceroni M, Camana C, Franciotta DM, et al. Serum and cerebrospinal fluid herpes simplex virus type 1 immunoglobulin $G$ and $M$ titers in four cases of herpes simplex encephalitis. Boll Soc Ital Biol Sper. 1990;66(12):1215-22.

10. Vandvik B, Vartdal F, Norrby E. Herpes simplex virus encephalitis: intrathecal synthesis of oligoclonal virus-specific IgG, IgA and IgM antibodies. J Neurol. 1982;228(1):25-38.

11. von Büdingen HC, Kuo TC, Sirota M, et al. B cell exchange across the blood-brain barrier in multiple sclerosis. J Clin Invest. 2012;122(12):4533-43.

12. Feng J, Fan S, Sun Y, et al. Study of B cell repertoire in patients with anti-N-methyl-D-aspartate receptor encephalitis. Front Immunol. 2020;11:1539.

13. Tsioris K, Gupta NT, Ogunniyi AO, et al. Neutralizing antibodies against West Nile virus identified directly from human B cells by single-cell analysis and next generation sequencing. Integr Biol (Camb). 2015;7(12):1587-97.

14. Bertoli D, Sottini A, Capra R, et al. Lack of specific Tand B-cell clonal expansions in multiple sclerosis patients with progressive multifocal leukoencephalopathy. Sci Rep. 2019;9(1):16605.

15. Alamyar E, Giudicelli V, Li S, Duroux P, Lefranc M-P. IMGT/HighV-QUEST: the IMGT web portal for immunoglobulin (Ig) or antibody and T cell receptor (TR) analysis from NGS high throughput and deep sequencing. Immunome Res. 2012;8:26.

16. Libbey JE, Fujinami RS. Adaptive immune response to viral infections in the central nervous system. Handb Clin Neurol. 2014;123:225-47.

17. Trück J, Eugster A, Barennes P, et al. Biological controls for standardization and interpretation of adaptive immune receptor repertoire profiling. Elife. 2021;10:e66274. 